\title{
Oral ulceration due to an antirheumatic drug (methotrexate): Report of a case
}

\author{
Yoshinori Jinbu $^{1}$, Yuri Obi ${ }^{1}$, Rumi Kawa ${ }^{1}$, Kaoru Ikeda ${ }^{1}$, Mikio Kusama ${ }^{1}$, Keiichi Tsukinoki ${ }^{2}$ \\ ${ }^{\prime}$ Department of Dentistry, Oral and Maxillofacial Surgery, Jichi Medical University, Shimotsuke, Japan \\ ${ }^{2}$ Department of Oral Diagnostic Science, Division of Pathology, Kanagawa Dental College, Yokosuka, Japan
}

Abstract: We report a case of oral ulceration that occurred during the course of rheumatoid arthritis treated with methotrexate. The patient was a 71-year-old man with oral ulceration of the floor of the mouth. The ulcer showed no induration, with a flat, clean surface and no bleeding. Biopsy revealed ulcer formation with marked inflammatory cell infiltration in the submucosa and dysplasia-like changes in epithelium. Topical steroids were ineffective. After reducing the dose of methotrexate, we observed that the lesion had epithelialized. The oral toxicity of low-dose methotrexate therapy is discussed.

[Oral Med Pathol 2008; 12: 97-99 doi: 10.3353/omp.12.97]

Key words: oral ulceration, methotrexate, adverse effect

Correspondence: Yoshinori Jinbu DDS, PhD, Department of Dentistry, Oral and Maxillofacial Surgery, Jichi Medical University, 3311-1 Yakushiji, Shimotsuke-shi, Tochigi 329-0498, Japan

Phone: +81-285-58-7390, Fax: +81-285-44-8669, E-mail: jinbu@jichi.ac.jp

\section{Introduction}

Methotrexate (MTX) is an antifolic agent with a wellestablished history of use in the treatment of various neoplastic diseases. Low-dose MTX (LDMTX) has been widely used for rheumatoid arthritis (RA) as a diseasemodifying antirheumatic drug (DMARD) (1). Since MTX was approved as an antirheumatic drug in 1999, use of LDMTX has become widespread in Japan. About 60,000 patients are estimated to be taking LDMTX for the treatment of RA (2). Many adverse effects of LDMTX have been reported (3-4), including stomatitis, although the frequency of this event is less common than with high-dose MTX therapy for cancer. As few reports have described stomatitis with LDMTX (5-6), detailed descriptions of the presentations and treatment for stomatitis due to LDMTX are lacking.

We report herein a case of oral mucosal ulceration that occurred during the course of RA treated with LDMTX.

\section{Case report}

The patient was a 71-year-old man with oral mucosal ulceration of the floor of the mouth and a 6-year history of RA. Medical history included RA, hypertension, prostatic hyperplasia and cardiac disease. He had been treated with MTX (8 mg/week), NSAIDs, corticosteroids, sulfa drugs, calcium-channel blockers, isosorbide, tranexamic acid and a vitamin compound. He complained of oral mucosal ulceration of the floor of the mouth without pain in May 2007. Although he was treated with corticosteroid ointment, ulceration did not improve. In June, he consulted an otolaryngologist who diagnosed denture stomatitis. However, no improvements were achieved with removal of the dentures. The patient then consulted another oral surgeon and underwent abrasion cytology. Pathological findings did not indicate malignancy.

Ulceration $(22 \times 18 \mathrm{~mm})$ of the left floor of the mouth with no induration was seen (Fig. 1). The surface of the ulceration was flat and clean with no bleeding. The ulcer

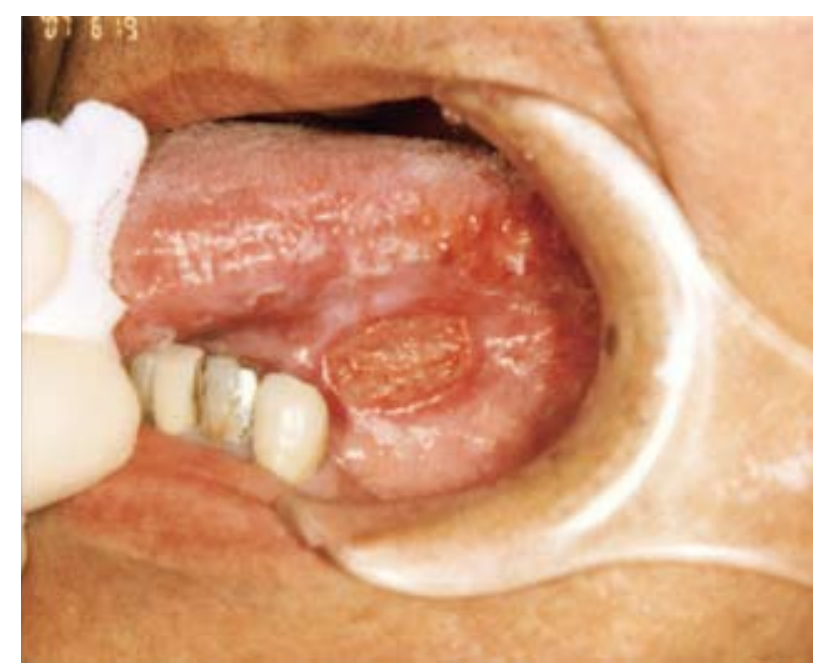

Fig. 1. Ulceration of the left floor of the mouth. The surface of the ulcer $(22 \times 18 \mathrm{~mm})$ was flat and clean with no bleeding. The ulcer margins were slightly raised but had no induration. 
margins were slightly raised. Blood examination revealed no abnormal values. Clinical diagnosis was chronic oral
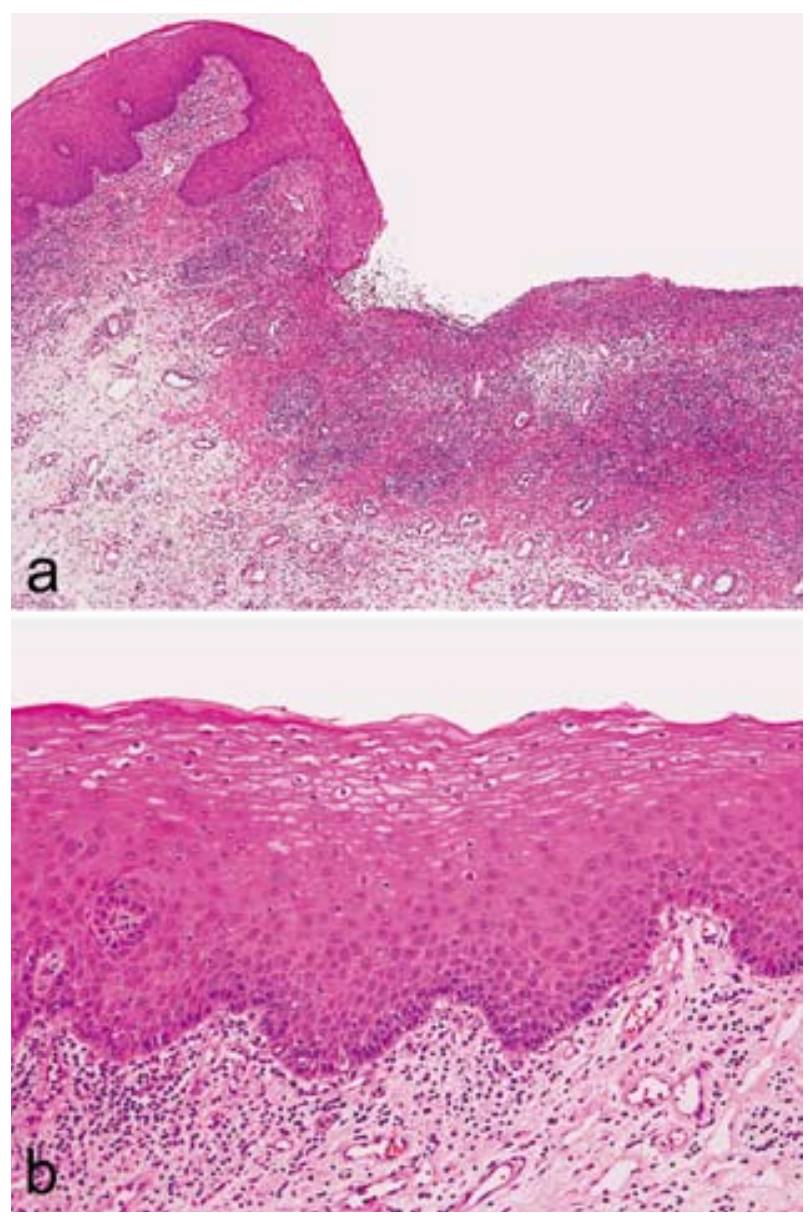

Fig. 2. Histopathology of oral ulcer. HE stain. (a) $\times 40$, (b) $\times$ 200. Ulcer formation with marked inflammatory cell infiltration in the submucosa. Epithelium surrounding the ulcer displayed focal dysplasia-like changes. A change toward spindle cell shapes was identified in the basal cell layer. In addition, although increased mitosis and N/C ratio were identified in the basal and parabasal cell layers, no loss of polarity was observed in the epithelium.

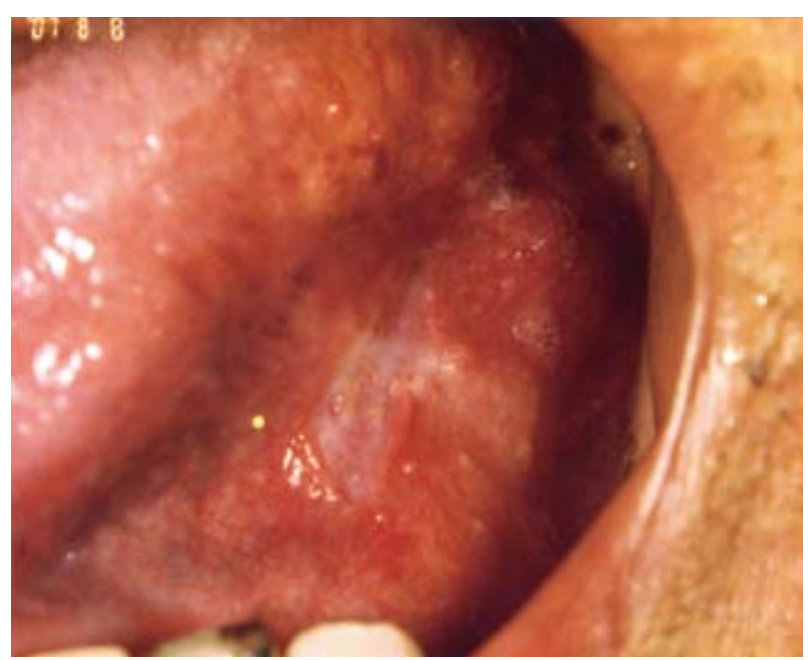

Fig. 3. Ameliorated ulcer after dose reduction. When the dose of MTX was reduced from $8 \mathrm{mg} /$ week to $2 \mathrm{mg} /$ week, oral ulceration greatly ameliorated and the lesion reepithelialized. ulceration of the floor of the mouth. As the ulcer was unchanged with administration of corticosteroid ointment for 1 week, oral biopsy was performed.

A section taken from the lesion showed ulcer formation with marked inflammatory cell infiltration in the submucosa. Epithelium surrounding the ulcer displayed focal dysplasialike changes. In particular, a change toward spindle cell shapes was identified in the basal cell layer. In addition, although increased mitosis and N/C ratio were identified in the basal and parabasal cell layers, no loss of polarity was observed in the epithelium (Fig. 2).

We suspected MTX as the responsible agent and contacted his physician. When the dose of MTX was reduced from $8 \mathrm{mg} /$ week to $2 \mathrm{mg} /$ week, oral ulceration greatly ameliorated and the lesion re-epithelialized (Fig. 3).

\section{Discussion}

Although LDMTX is able to suppress inflammatory activity and attenuate the disease process in RA, many adverse effects have been reported $(7,8)$. As general adverse effects, gastrointestinal toxicity (nausea, vomiting, abdominal discomfort, anorexia, dyspepsia, diarrhea), hepatotoxicity (hepatitis, fibrosis, cirrhosis), myelosuppression (leukocytopenia, thrombocytopenia, pancytopenia), hyperhomocysteinemia, hypersensitivity causing pulmonary toxicity, central nervous system events (headaches, depression) and osteoporosis have been described. Severe oral toxicity is a common feature of high-dose MTX therapy for the treatment of malignant disease. Oral toxicity in LDMTX is less common, although frequencies of oral mucosal lesions have varied widely with different investigators, from $1 \%$ to $64 \%$. According to Kalantzis et al. (6), the mean frequency of stomatitis with LDMTX is $14 \%$.

As oral symptoms, a variety of oral lesions have been described, including recurrent ulceration, bullae, lichenoid reaction, non-healing ulcer and lymphoma-like lesion (6). Some oral lesions, particularly widespread geographic ulcer, are said to occur as a prodromal symptom of bone marrow suppression (9). Detailed descriptions of LDMTX-induced oral symptoms have not been well reported in the dentistry and oral medicine area. In the present case, non-healing ulcer of the floor of the mouth with no induration was observed. The surface of the ulceration was flat and clear with no bleeding and enclosed, raised margins. These characteristic features are clearly different from stomatitis induced by high-dose MTX therapy and may represent diagnostic features allowing differentiation from malignant lesions.

MTX is a bicarboxylic acid and folic acid analog that inhibits dihydrofolate-reductase (DHFR) (10). Inhibition of DHFR prevents reduction of folic acid to tetrahydrofolates and reduces nucleotide synthesis and homocysteine remethylation. In addition, MTX directly inhibits other enzymes in the folate pathway of pyrimidine synthesis and purine synthesis (11). MTX thus inhibits synthesis of AMP, GMP, DMA and RNA and inhibits the cell cycle. MTX also causes release of adenosine, which has anti-inflammatory and 
immunosuppressive effects (10). Adenosine reduces neutrophil adherence and neutrophil-mediated tissue damage. MTX reduces secretion of interleukins, tumor-necrosis factors and interferons in inflamed synovium and causes apoptosis of activated T-lymphocytes (11-12).

Adverse effects of LDMTX have been divided into 3 major groups (13). The first group, including direct gastrointestinal and bone marrow toxicity, are dosedependent effects. These effects are mediated by folate antagonism and are the most common. The second group includes idiosyncratic or allergic reactions, such as pneumonitis. The third group includes effects of long-term treatment caused mainly by hyper-homocysteinemia. Oral toxicity is considered a dose-dependent effect.

Very few reports have provided histological analysis of oral lesions induced by LDMTX therapy $(5-6,14)$. Kalantzis et al. (6). reported histology for 7 cases with oral lesions following LDMTX treatment. Of these, 3 cases showed nonspecific ulcers. In the other 4 cases, histology showed fibroepithelial polyp, EBV-related lymphoproliferative lesion, pseudodysplasia of surrounding mucosa, lichenoid appearance and Wegener's granulomatosis or lymphoma, respectively. In the epithelia of these lesions, histological characteristics similar to folate deficiency were reported, such as anisonucleosis, hyperchromatin, basal cell disorganization and suprabasal mitosis (6). In the present case, histology revealed dysplasia-like changes in epithelium and a change toward spindle cell shapes in the basal cell layer. Increased mitosis and N/C ratio were observed in the basal and parabasal cell layers.

For the treatment of oral lesions resulting from LDMTX therapy, use of traditional agents such as topical analgesics or steroids, mouthwash and covering agents have been reported (5). However, these agents appear to provide only symptomatic relief. In the present case, topical steroids were completely ineffective and the oral lesion healed only after dose reduction of MTX. Kalantzis et al. (6) suggested treatment procedures for oral lesions due to LDMTX therapy comprising folate supplementation, MTX cessation, dosereduction of MTX, excision of the lesion, switching to another DMARD, combination regime (MTX plus other DMARDs) and combination or substitution with newer biological agents.

Dentists are increasingly likely to encounter patients taking MTX for treatment of RA and so should be aware of clinical and histopathological findings of oral complications caused by MTX therapy.

\section{References}

1. O'Dell JR. Methotrexate use in rheumatoid arthritis. Rheum Dis Clin North Am 1997; 23: 779-96.

2. Suzuki Y. Methotrexate for the treatment of rheumatoid arthritis in Japan-Much more still remains to be resolvedNippon Rinsho 2002; 60: 2331-8.

3. Furst DE, Koehnke R, Burmeister LF, Kohler J, Cargill I. Increasing methotrexate effect with increasing dose in the treatment of resistant rheumatoid arthritis. J Rheumatol 1989; 16: 13-20.

4. Schnabel A, Gross WL. Low-dose methotrexate in rheumatic disease: efficacy, side effects, and risk factors for side effects. Semin Arthritis Rheum 1994; 23: 310-27.

5. Deeming GMJ, Collingwood J, Pemberton MN. Methotrexate and oral ulceration. Br Dent J 2005; 198: 83-5.

6. Kalantzis A, Marshman Z, Falconer DT, Morgan PR, Odell EW. Oral effects of low-dose methotrexate treatment. Oral Surg Oral Med Oral Pathol Oral Radiol Endod 2005; 100: 52-62.

7. Mielants H, Veys EM, Van des Straeten C, Ackerman C, Goemaere S. The efficacy and toxicity of a constant low dose of methotrexate as a treatment for intractable rheumatoid arthritis: an open prospective study. J Rheumatol 1991; 7: 978-83.

8. McKendry RJ, Dale P. Adverse effects of low dose methotrexate therapy in rheumatoid arthritis. J Rheumatol 1993; 11: 1850-6.

9. Kuga Y. How should methotrexate be used safe [sic] in patients with rheumatoid arthritis[sic]. Bone Joint Ligament 2005; 18: 521-7.

10. van Ede AE, Laan RF, Blom HJ, De Abreu RA, van de Putte LB. Methotrxate in rheumatoid arthritis: an update with focus on mechanisms involved in toxicity. Semin Arthritis Rheum 1998; 5: 277-92.

11. Genestier L, Paillot R, Quemeneur L, Izeradjene K, Revillard JP. Mechanisms of action of methotrexate. Immunopharmacology 2000; 2: 247-57.

12. Cutolo M, Sulli A, Pizzorni C, Seriolo B, Straub RH. Anti inflammatory mechanisms of methotrexate in rheumatoid arthritis. Ann Rheum Dis 2001; 8: 729-35.

13. McKendry RJ. The remarkable spectrum of methotrexate toxicities. Rheum Dis Clin North Am 1997; 23: 939-54.

14. Iwamoto O, Nakamura M, Koga C, Kusukawa J. A case of oral mucous membrane ulcer cased by disease modifying antirheumatis drug (DMARD). J Jpn Oral Muco Membr 2007; 13: 43-6. 